\title{
A spectroscopic and photometric study of the interacting binary and double period variable HD 170582
}

\author{
R. E. Mennickent ${ }^{1}$, G. Djuraševići ${ }^{2}$, M. Cabezas ${ }^{1}$, A. Cséki ${ }^{2}$, \\ J. Rosales ${ }^{1}$, E. Niemczura ${ }^{3}$, I. Araya ${ }^{4}$ and M. Curé ${ }^{4}$ \\ ${ }^{1}$ Departamento de Astronomía, Universidad de Concepción, Chile, \\ email: rmennick@astroudec.cl \\ ${ }^{2}$ Astronomical Observatory, Belgrade, Serbia, ${ }^{3}$ Astronomical Institute, Wrocław University, \\ Poland, ${ }^{4}$ Instituto de Física y Astronomía, Facultad de Ciencias, U. de Valparaíso, Chile
}

\begin{abstract}
We present a spectroscopic and photometric study of the interacting binary and Double Period Variable HD 170582 based on the analysis of the ASAS $V$-band light curve and our high-resolution spectra mostly obtained with CHIRON spectrograph at the $1.5 \mathrm{~m}$ CTIO telescope.
\end{abstract}

Keywords. binaries: eclipsing, binaries: close, stars: mass-loss, stars: fundamental parameters.

\section{On the poorly known binary HD 170582}

HD 170582 (ASAS ID 183048-1447.5, $\alpha_{2000}=18: 30: 47.5, \delta_{2000}=-14: 47: 27.8$, $V=9.66 \mathrm{mag}, B-V=0.41 \mathrm{mag}$, spectral type A $9 \mathrm{~V})$, is a poorly studied binary star catalogued semi-detached eclipsing binary with orbital period 16.8599 days in the ASAS catalogue (Pojmanski 1997). It is located in the region of the cool molecular cloud L379 and is characterized by a long photometric cycle of 536 days. It is the longest-period member of the Galactic Double Period Variables (DPVs, Fig. 1). DPVs are intermediate mass interacting binaries showing a long photometric cycle lasting about 33 times the orbital period, which has been interpreted as cyclic episodes of mass loss (Mennickent et al. 2003, 2008, 2012a,b; Poleski et al. 2010).

\section{Light curve model and spectroscopic analysis}

Based on the study of the ASAS $V$-band light curve we determine an improved orbital period of $16.87177 \pm 0.02084$ days and a long period of 587 days. We disentangled the light curve into an orbital part, determining ephemerides and revealing orbital ellipsoidal variability with unequal maxima, and a long cycle, showing quasi-sinusoidal changes with $V$-band amplitude $0.1 \mathrm{mag}$. From the analysis of 136 CHIRON/CTIO high-resolution optical spectra, the model of the $V$-band ASAS light curve and the fit of the spectral energy distribution, we determine the physical parameters for the stars and the circumprimary disc, the distance to the system and general system dimensions, reddening and metallicity. For the light curve model we use the code described by Djurašević (1992) solving the inverse problem for the Roche model with an accretion disk around the more-massive (hotter) gainer. The best model for the donor star is characterized by $T_{2}=8000 \pm 125 \mathrm{~K}$, $\log g_{2}=1.5 \pm 0.25$ and projected rotational velocity $v_{2 \mathrm{r}} \sin i=44 \pm 4 \mathrm{~km} \mathrm{~s}^{-1}$. Assuming synchronous rotation for the donor we obtain a mass ratio of $q=0.21$. The disc contributes about $35 \%$ to the system luminosity at the $V$-band. Two extended regions 


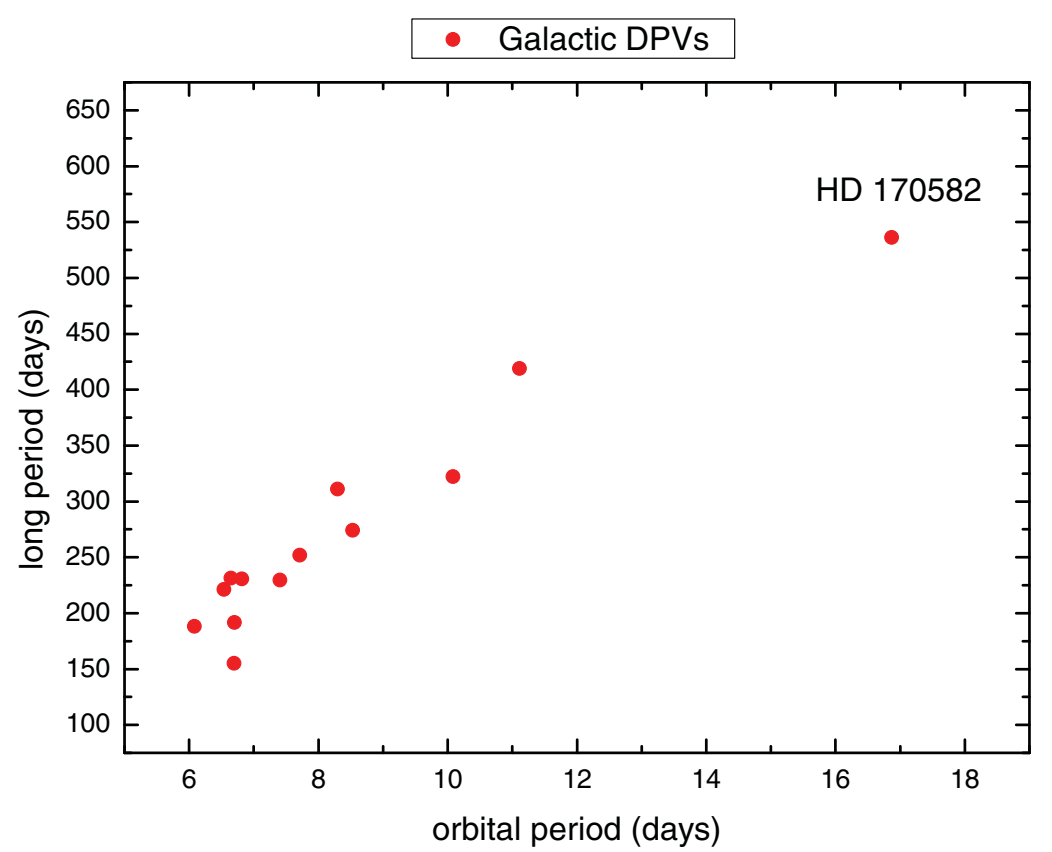

Figure 1. Galactic Double Period Variables according to Mennickent et al. (2012a).

located at opposite sides of the disc rim, and hotter than the disc by $67 \%$ and $46 \%$, fit the light curve asymmetries. These structures can be attributed to shocks produced by disc gas dynamics and gas stream interaction. The system is seen under inclination 67 degree and it is found at a distance of $238 \mathrm{pc}$. We discuss the double line nature of HeI 5875; two absorption components move in anti-phase during the orbital cycle. A possible origin for one of these component is a place near the stream/disc interaction region. We find that HD 170582 is one of the systems showing a discrepancy between the color excess obtained from diffuse interstellar bands and that obtained from the analysis of the spectral energy distribution. This might be attributed to the influence of circumstellar matter. This study of HD 170582 will help to understand the class of interacting binaries Double Period Variables. A full study has been submitted for publication.

\section{References}

Djurašević, G. 1992, Ap\&SS 197, 17

Mennickent, R. E., Djurašević, G., Kołaczkowski, Z., \& Michalska, G. 2012a, MNRAS 421, 862

Mennickent, R. E., Kołaczkowski, Z., Djurašević, G., et al. 2012b, MNRAS 427, 607

Mennickent, R. E., Kołaczkowski, Z., Michalska, G., et al. 2008, MNRAS 389, 1605

Mennickent, R. E., Pietrzyński, G., Diaz, M., \& Gieren, W. 2003, A $\& A$ 399, L47

Pojmanski, G. 1997, AcA 47, 467

Poleski, R., Soszyñski, I., Udalski, A., et al. 2010, AcA 60, 179 